\title{
Evaluation of a Hybrid Boltzmann-Continuum Method for High Speed Nonequilibrium Flows
}

\author{
Jonathan M. Burt, ${ }^{1}$ Timothy R. Deschenes, ${ }^{2}$ and Iain D. Boyd ${ }^{3}$ \\ Department of Aerospace Engineering \\ University of Michigan, Ann Arbor, MI 48109 \\ Eswar Josyula ${ }^{4}$ \\ U.S. Air Force Research Laboratory \\ Wright-Patterson Air Force Base, OH 45433
}

\begin{abstract}
A series of monatomic gas flows over a cylinder, with a freestream Mach number of 4 and a range of Knudsen numbers, are used to evaluate the unified flow solver (UFS) code. The UFS code combines several compressible gas flow simulation schemes for application to flow problems involving a wide range of Knudsen number $(\mathrm{Kn})$ regimes, and features capabilities for strong coupling between low-Kn and high-Kn schemes along with automatic tree-based grid adaptation. UFS simulation results are compared with results from simulations which employ other codes intended for the same class of problems, and good agreement is generally found. Areas identified for improvement in UFS include calculation of surface quantities and numerical performance of the UFS Boltzmann equation solver.
\end{abstract}

\section{Introduction}

A variety of gas flow problems are characterized by a wide range of local Knudsen number (Kn) regimes, with strong translational nonequilibrium in some portions of the flowfield and near-equilibrium velocity distributions in other flowfield regions. (The Knudsen number, which quantifies the level of translational nonequilibrium in a gas flow, is defined as the ratio of the mean free path to some characteristic length scale based on boundary geometry or gradients.) These problems include hypersonic aerodynamics flows, such as those around atmospheric entry vehicles; rocket exhaust flows at high altitudes; spacecraft gas venting; shock-boundary layer interactions and other flows for which internal shock structure is important; and subsonic flows, such as those within MEMS devices, which involve either very small length scales or low gas density.

For accurate simulation of high-Kn rarefied regions within these flows, translational nonequilibrium effects must be considered, and either a particle scheme such as the direct simulation Monte Carlo (DSMC) method ${ }^{1}$ or a direct simulation method for the governing Boltzmann equation ${ }^{2}$ is required. While these methods can be applied to low$\mathrm{Kn}$ regions as well, they tend to be far more computationally expensive than continuum computational fluid dynamics (CFD) methods based on the Navier-Stokes equations while offering the same degree of accuracy in lowKn regimes. Thus, an optimal combination of accuracy and efficiency for simulation of mixed rarefied-continuum flows generally requires integration of rarefied and continuum schemes within a coupled numerical framework. In this hybrid approach, the simulation domain is divided into rarefied and continuum flow regions through evaluation of a continuum breakdown parameter. DSMC or Boltzmann solver calculations are performed in rarefied regions, a continuum CFD method is applied in continuum regions, and coupling routines are used to exchange conserved quantities or other flow information across continuum breakdown boundaries.

In recent years, most work on algorithm development for mixed rarefied-continuum flow simulations has focused on hybrid CFD-DSMC techniques. ${ }^{3-8}$ This type of technique has been shown to preserve the physical accuracy of DSMC in high-Kn regions, while offering the numerical efficiency of a CFD Navier-Stokes solver in low-Kn regions. However, the inherent statistical scatter in DSMC can make coupling between the two methods difficult, and much of the focus in hybrid DSMC-CFD development has been on scatter reduction to avoid

\footnotetext{
${ }^{1}$ Post-doctoral research fellow, AIAA member.

${ }^{2}$ Graduate student, AIAA student member.

${ }^{3}$ Professor, AIAA associate fellow.

${ }^{4}$ Senior research aerospace engineer, AIAA associate fellow.
} 
inaccurate or unstable CFD calculations. The scatter problem becomes particularly severe in simulations of highly unsteady flows, subsonic flows, or flow problems (such as those involving gas radiative emission or chemistry) for which accurate characterization is desired for the low-probability tails of the velocity distribution. In these cases, a very large number of DSMC particles may be required for sufficient scatter reduction, and a hybrid CFD-DSMC simulation can be prohibitively expensive.

In one alternative hybrid scheme, a set of direct simulation methods for the Boltzmann equation are coupled to kinetic methods for the compressible Navier-Stokes or Euler equations. ${ }^{9-13}$ This hybrid scheme, implemented in the unified flow solver (UFS) code, is not prone to the DSMC statistical scatter problem which may be considered the main drawback in CFD-DSMC techniques. Instead of using a collection of particles to represent the gas velocity distribution within rarefied regions, as in DSMC, nonequilibrium velocity distributions in UFS are modeled using a Cartesian grid in velocity space. Discrete approximations of the advection and collision terms in the Boltzmann equation are employed to update the probability density at grid points in both velocity and physical space, and coupling across continuum breakdown boundaries is performed through two-way information exchange during each simulation time step. The UFS code is based on the open source framework of the Gerris flow solver, ${ }^{14}$ and includes capabilities for automatic binary tree-based Cartesian grid adaptation. Solid 2D or 3D objects are easily integrated within the simulated flowfield through the use of cut-cell boundaries, and additional procedures allow optimized load balancing for parallel domain decomposition. Continuum breakdown evaluations are periodically performed during a UFS simulation, for automatic assignment of each grid cell to either continuum or rarefied domains. Performance improvements, relative to DSMC or full Boltzmann solutions, are realized in UFS through the use of continuum CFD methods where appropriate, and through initialization of rarefied domain calculations with (at least partially converged) continuum method solutions. In comparison with DSMC, reduced simulation expense has been demonstrated in UFS simulations of a nozzle/plume expansion flow, ${ }^{11}$ and comparable expense to DSMC has been observed in UFS simulations of a hypersonic blunt body flow. ${ }^{12}$

In the current effort, the UFS scheme is applied to a set of high Mach number rarefied/continuum flows over a cylinder, and is evaluated for accuracy and efficiency through comparison with DSMC and CFD Navier-Stokes methods. As previous evaluations of UFS have been carried out for a variety of flow problems, new objectives here include performing a more comprehensive comparison for accuracy and efficiency with both DSMC and CFD, and highlighting limitations, drawbacks or areas to prioritize for future development of the UFS code.

In the following sections, simulation setup and input parameters are described for a number of simulations performed on either UFS or other codes which are used for comparison. The computational expense of various simulations is then discussed, and a general efficiency comparison is made between UFS and the other codes employed in this study. Next, detailed results are presented. The accuracy of UFS simulation results is assessed by comparing surface flux coefficients, contour lines and variation along the stagnation streamline in selected flow properties. Drag coefficients computed from these simulations are then plotted as a function of global Knudsen number, in order to further assess accuracy of UFS simulations, compare with available experimental data, and evaluate the dependence of drag on the cylinder surface temperature. Finally, results are summarized and positive characteristics, potential problems and possible directions for future work involving UFS are discussed.

\section{Simulation Setup}

As the basis for a series of test problems, we consider a flow of argon over a cylinder with a freestream Mach number of 4 . The hard sphere collision model is used, so that collision cross sections are independent of the relative speed between colliding particles and viscosity varies as the square root of temperature. ${ }^{1}$ Diffuse reflection with full thermal accommodation to the wall temperature is assumed on the cylinder surface, and the temperature at the wall is set to 1.2 times the freestream temperature. (In simulations for which NS calculations are used over the entire flowfield, a no-slip wall boundary condition is employed.) Simulations are performed for global Knudsen numbers $\mathrm{Kn}$ (the ratio of the freestream mean free path to cylinder diameter) of $0.3,0.03$ and 0.003 using various models in UFS. For comparison, additional simulations are performed using the DSMC code MONACO ${ }^{15}$ and the NavierStokes CFD code LeMANS. ${ }^{16}$

The $\mathrm{Kn}=0.3$ case is representative of a highly rarefied flow, and is simulated using numerical solutions to the Boltzmann equation and the Bhatnagar-Gross-Krook (BGK) equation. ${ }^{2}$ A Navier-Stokes (NS) simulation of this flow is also performed using LeMANS. In the $\mathrm{Kn}=0.03$ case, large portions of the flowfield are within both rarefied and continuum regimes, and hybrid schemes involving coupled rarefied and continuum flow calculations should give the best combination of accuracy and efficiency. This flow is simulated using UFS with the following options: BGK, NS, hybrid BGK-NS and hybrid BGK-Euler. The accuracy of all UFS results at Kn $=0.3$ and 0.03 is assessed through comparison with results from simulations using the DSMC code MONACO. The Kn $=0.003$ case, 
for which only small portions of the flowfield are in significant thermal nonequilibrium, is used to evaluate the NS solver in UFS by comparing UFS NS results with those from a NS simulation using the LeMANS CFD code. To demonstrate the expected inaccuracy for NS simulations at $\mathrm{Kn}=0.3$ and 0.03 , LeMANS results are also generated for these cases.

MONACO simulations in this study employ unstructured grids which are automatically adapted to the local mean free path using a grid generation utility AFMGEN, and dynamically adaptive DSMC subcells are used to assure sufficiently small mean collision separation values. Spatially uniform time step intervals in MONACO are carefully set to meet DSMC requirements, ${ }^{1}$ and numerical weight values are dynamically adapted in each cell to meet standard DSMC guidelines (at least 20 particles per cell) while avoiding any efficiency reduction due to excessive particle populations. LeMANS simulations use a finite volume implicit second-order modified version of the Steger-Warming flux vector splitting scheme, ${ }^{17}$ which is less dissipative in boundary layers but switches back to the original form in the vicinity of strong shocks.

In hybrid UFS simulations, a continuum breakdown parameter based on characteristic length scales for velocity and pressure gradients is used to assign flowfield regions to continuum or rarefied domains. Rarefied regions are identified by periodically comparing values of a breakdown parameter $S_{N S}$ to a threshold value of 0.1 . Following Ref. 9, the breakdown parameter is defined as

$$
S_{N S}=\lambda \sqrt{\left(\frac{\nabla p}{p}\right)^{2}+\left[\left(\frac{\partial u}{\partial x}\right)^{2}+\left(\frac{\partial v}{\partial y}\right)^{2}+\left(\frac{\partial w}{\partial z}\right)^{2}\right] /\left(u^{2}+v^{2}+w^{2}\right)}
$$

where $\lambda$ is the mean free path, $p$ is the pressure, and $(u, v, w)$ is the bulk velocity. UFS calculations in the rarefied domain utilize either a direct numerical solution to the discretized Boltzmann equation or the BGK approximation to the Boltzmann equation, and the second-order kinetic NS method of $\mathrm{Xu}^{18}$ is used in the continuum domain.

Spatial and velocity grid independence and solution convergence is verified for all simulations. The full Boltzmann calculation uses a velocity grid of $40 \times 40 \times 20$ points, in the $\mathrm{x}, \mathrm{y}$ and $\mathrm{z}$ directions respectively, while BGK calculations are performed on a grid of $40 \times 40 \times 2$ points in velocity space. Both Boltzmann and BGK simulations take advantage of velocity distribution symmetry along the $\mathrm{z}$ axis, in order to reduce the number of velocity grid points.

\section{Efficiency comparison}

Table 1 shows a summary of numerical parameters for 12 different UFS, DSMC, and LeMANS simulations performed as part of this study. Computational expense is comparable for UFS NS and LeMANS simulations at Kn $=0.003$, with fewer cells but more iterations in the UFS simulation. Note that the difference in cell count between these two simulations is due in large part to the use of gradient-based mesh adaptation in UFS, which allows for larger cells in low-gradient regions. This difference is partially offset by the use of stretched (i.e. high aspect ratio) cells near the cylinder surface in the LeMANS simulation; UFS requires that square cells be used, so far more cells are needed near the surface for similar grid resolution in the surface-normal direction. At $\mathrm{Kn}=0.03$ considerable efficiency gains are found in UFS NS calculations relative to LeMANS, presumably due to a larger difference in cell count between UFS and LeMANS at this higher Kn value.

As shown in the table, the DSMC simulation for $\mathrm{Kn}=0.03$ is almost equally expensive as the corresponding BGK simulation, while DSMC is only $20 \%$ as expensive as BGK at $\mathrm{Kn}=0.3$. As expected, efficiency gains are found in the hybrid BGK-NS and BGK-Euler simulations in comparison to the full BGK simulation at $\mathrm{Kn}=0.03$, although these gains are smaller than $15 \%$ for both hybrid simulations. Given the added complexity of hybrid scheme implementation and the fact that BGK and NS calculations should have comparable accuracy within continuum regions, the similar level of computational expense for BGK and hybrid UFS simulations at $\mathrm{Kn}=0.03$ seems to indicate that hybrid techniques are not preferable for this case.

The most surprising finding shown in the table is the enormous difference in expense between DSMC and Boltzmann simulations at $\mathrm{Kn}=0.3$. Here the DSMC simulation requires less than $0.2 \%$ of the CPU time needed for the Boltzmann simulation, which corresponds to a difference of over two orders of magnitude in computational expense. As discussed above, a direct numerical solution of the Boltzmann equation should have similar accuracy to DSMC, and holds potential advantages to DSMC in simulating a variety of rarefied or multiscale gas flows (particularly unsteady or subsonic flows, flows for which the tails of the velocity distribution must be characterized with high precision, or cases for which strong coupling with a NS solver is desired). Still, for this particular case it 
seems reasonable to conclude that DSMC simulation is preferable to simulation using the UFS Boltzmann solver, assuming that both simulations can provide comparable accuracy.

\begin{tabular}{|l|l|l|l|l|l|}
\hline $\begin{array}{l}\text { Simulation } \\
\text { method }\end{array}$ & $\begin{array}{l}\text { Knudsen } \\
\text { number }\end{array}$ & Cells & Iterations & Processors & CPU hours \\
\hline $\begin{array}{l}\text { Boltzmann } \\
\text { (UFS) }\end{array}$ & 0.3 & 6515 & 10000 & 16 & 1929.4 \\
\hline BGK (UFS) & 0.3 & 6605 & 10000 & 8 & 18.5 \\
\hline LeMANS NS & 0.3 & 14261 & 9500 & 8 & 4.47 \\
\hline $\begin{array}{l}\text { DSMC } \\
\text { (MONACO) }\end{array}$ & 0.3 & 3735 & $\begin{array}{l}100000 \\
(50000 \text { sampling steps) }\end{array}$ & 8 & 3.75 \\
\hline BGK (UFS) & 0.03 & 23208 & 20000 & 8 & 66.5 \\
\hline BGK-NS (UFS) & 0.03 & 10490 (582 NS) & 20000 & 8 & 60.0 \\
\hline $\begin{array}{l}\text { BGK-Euler } \\
\text { (UFS) }\end{array}$ & 0.03 & $11271(874$ Euler) & 20000 & 8 & 56.5 \\
\hline UFS NS & 0.03 & 19719 & 20000 & 8 & 9.88 \\
\hline LeMANS NS & 0.03 & 101959 & 11700 & 8 & 41.3 \\
\hline $\begin{array}{l}\text { DSMC } \\
\text { (MONACO) }\end{array}$ & 0.03 & 65189 & $\begin{array}{l}200000 \\
(100000 \text { sampling steps) }\end{array}$ & 8 & 64.3 \\
\hline UFS NS & 0.003 & 72446 & 40000 & 8 & 50.9 \\
\hline LeMANS NS & 0.003 & 101959 & 13500 & 8 & 48.0 \\
\hline
\end{tabular}

Table 1. Comparison of simulation parameters and CPU time for UFS, MONACO and LeMANS simulations.

\section{Comparison of simulation results for $\mathrm{Kn}=0.3$}

Figure 1 shows computational grids used in UFS BGK and MONACO DSMC simulations for the $\mathrm{Kn}=0.3$ case. Note that grids used in UFS calculations are adapted over the course of a simulation, and the BGK grid shown in Fig. 1 is taken from steady state results after a total of 10,000 iterations. As observed in the figure, cell sizes in both the freestream and farfield wake regions are similar in the two simulations, although significant differences are found in cell size within shock layer and nearfield regions. These discrepancies in cell size can be attributed to differences in grid refinement criteria between MONACO and UFS: MONACO cells are refined to the local mean free path, while grid refinement in UFS is based on density and velocity gradients.

In Fig. 2, contours are shown for the maximum gradient length local Knudsen number $\mathrm{Kn}_{\mathrm{GLL}-\max }$, as calculated from DSMC simulation results at $\mathrm{Kn}=0.3$. The parameter $\mathrm{Kn}_{\mathrm{GLL}-\max }$ is computed as the ratio of the local mean free path to the smallest length scale based on gradients in bulk velocity, density or temperature. The Navier-Stokes equations are usually assumed valid for $\mathrm{Kn}_{\mathrm{GLL}-\max }<0.05 .{ }^{19}$ As shown in the figure, continuum breakdown occurs over nearly the entire flowfield.

Figure 3 shows contours of bulk velocity magnitude from MONACO, UFS and LeMANS simulations at Kn $=$ 0.3. All values in the legend are normalized by the freestream velocity. Results from Boltzmann, BGK and DSMC simulations are shown in the upper half of the figure, while LeMANS NS and DSMC simulation results are compared in the lower half. As expected, both Boltzmann and BGK solutions show good overall agreement with DSMC, while the BGK results give slightly worse agreement with DSMC than Boltzmann simulation results. Poor agreement is observed between DSMC and NS results through much of the flowfield, which is consistent with the high level of continuum breakdown found in Fig. 2. Most of the discrepancy between DSMC and NS results can be attributed to the failure of assumptions underlying the Navier-Stokes equations; in regions of significant nonequilibrium, as indicated in Fig. 2, gradient-based diffusive transport approximations tend to break down, and the gas velocity distribution function cannot be accurately approximated using small perturbations from equilibrium. Both gradient transport and small perturbation assumptions are used in the NS calculations.

Figures 4 and 5 show contours of normalized density and temperature, respectively, for all simulations at $\mathrm{Kn}=$ 0.3. Similar trends are found in both figures as in Fig. 3. In particular, good overall agreement is found between DSMC and Boltzmann simulation results, with only slightly worse agreement between DSMC and BGK. A significantly broader bow shock region, as indicated by temperature contours, is observed in BGK results than in results from either DSMC or Boltzmann simulations. As in Fig. 3, poor agreement is found between DSMC and NS results over nearly the entire flowfield in both Figs. 4 and 5.

Figures 6, 7 and 8 show the variation along the stagnation streamline in the normalized bulk velocity, density and temperature, respectively, from the $\mathrm{Kn}=0.3$ simulations. In all three figures we find very good agreement 
between Boltzmann and DSMC results, with only a small overestimate relative to DSMC in the shock thickness based on either velocity or temperature. Note that, as expected at this high global $\mathrm{Kn}$, the shock is represented in all simulations as a broad region of smooth compression, and (particularly in Fig. 7) the shock, shock layer, and boundary layer cannot be easily distinguished. Noticeable differences are observed, however, between DSMC, BGK and NS results in the level of diffusive transport: A significantly wider shock region is found in the BGK simulation than in the DSMC simulation, while the NS simulation gives a narrower shock and larger near-wall boundary layer gradients.

In Fig. 9, the nondimensional surface pressure coefficient (based on a vacuum reference pressure) is plotted along the cylinder surface for simulations at $\mathrm{Kn}=0.3$. The angle $\theta$, used for the horizontal axis, is defined such that front and rear stagnation points are located at $0^{\circ}$ and $180^{\circ}$, respectively. As expected, excellent agreement is found over the entire surface between Boltzmann and DSMC results, and small but noticeable disagreement between DSMC and BGK occurs for $\theta>120^{\circ}$. Note the "stair step" pattern in the UFS simulation results, which is presumably caused by the use of a cut cell boundary condition along the cylinder surface. Implementation of an alternate immersed boundary method, which is currently underway, is anticipated to reduce or eliminate this problem. Results from the NS simulation agree well with DSMC only for $\theta<60^{\circ}$, with considerable qualitative disagreement near $180^{\circ}$. Similar trends are observed in Fig. 10, which shows the corresponding variation in the surface friction coefficient. In this figure, very good agreement is found between DSMC, BGK and Boltzmann results over the entire surface, while the friction coefficient is overestimated in the NS simulation by approximately a factor of three at $0^{\circ}$ and by over three orders of magnitude at $180^{\circ}$.

Figure 11 shows the surface heat transfer coefficient for the $\mathrm{Kn}=0.3$ simulations. While surprisingly good agreement (within about 20\%) is found between NS and DSMC results at the front stagnation point, the level of disagreement increases rapidly with $\theta$, and the NS surface heat transfer is overestimated by around three orders of magnitude at $\theta=180^{\circ}$. Note that somewhat better agreement between NS and DSMC would be expected in Fig. 11, as well as in Figs. 9 and 10, if the NS wall boundary condition included consideration of velocity slip and temperature jump effects.

As in Figs. 9 and 10, good overall agreement is found in Fig. 11 over the entire cylinder surface between DSMC, BGK and Boltzmann simulation results. Slightly larger discrepancies are found between DSMC and BGK than between DSMC and Boltzmann results, with a noticeable underestimate in BGK surface heat transfer along much of the afterbody and a small corresponding overestimate along the forebody. Although recently published UFS results have shown an overestimate in UFS heat transfer by approximately a factor of two, ${ }^{20}$ the authors were made aware of a normalization factor of 0.5 in UFS nondimensional heat transfer values which has been applied to values in Fig. 11 and which seems to provide far better agreement with DSMC. With this normalization factor, the overestimate relative to DSMC in stagnation point heat flux is only about 3.8\% for the Boltzmann simulation and $9.5 \%$ for the BGK simulation.

Much of the error observed in Boltzmann and BGK values, as well as the artificial "stair step" pattern in these values, is likely due to the cut boundary condition, and is expected to be eliminated through the use of the immersed boundary method in place of cut cells. Further error in surface heat transfer from Boltzmaan and BGK simulations may be due to insufficient mesh refinement near the surface, although comparable cell sizes are used in these simulations as in the DSMC simulation and it seems unlikely that mesh resolution accounts for most of the discrepancy.

\section{Comparison of simulation results for $\mathrm{Kn}=\mathbf{0 . 0 3}$}

Figure 12 shows meshes used in UFS BGK and DSMC simulations for the $\mathrm{Kn}=0.03$ case. As in Fig. 1 , differences in local refinement levels are attributed to the fact that DSMC cell size is adapted to the mean free path, while UFS mesh adaptation is based on density and velocity gradients. For both simulations, the shock position is clearly indicated by a sudden increase in mesh refinement levels upstream of the cylinder.

Figure 13 is a contour plot of the maximum gradient length local Knudsen number $\mathrm{Kn}_{\text {GLL-max }}$ from the DSMC simulation at $\mathrm{Kn}=0.03$. In comparing this figure with Fig. 2, we find that continuum breakdown (defined by $\mathrm{Kn}_{\mathrm{GLL}-\max }>0.05$ ) occurs over a far smaller portion of the flowfield than for the $\mathrm{Kn}=0.3$ case. For the present case, continuum breakdown is limited to a region surrounding the bow shock, the forebody boundary layer, and a large portion of the wake. Figure 14 shows the boundaries between NS and BGK domains, at steady state, in a hybrid BGK-NS simulation of the $\mathrm{Kn}=0.03$ case. These boundaries, along which the right side of Eq. (1) is equal to a cutoff value of 0.1, correspond reasonably well to continuum breakdown boundaries shown in Fig. 13. Significant differences between domain boundaries in Fig. 14 and the 0.05 contour line in Fig. 13 are found only in the wake region. 
In Fig. 15, contours of normalized bulk velocity magnitude are shown for several different simulations at $\mathrm{Kn}=$ 0.03. Results are displayed for BGK, hybrid BGK-NS, hybrid BGK-Euler, DSMC, UFS NS and LeMANS NS simulations. Contour lines from both UFS NS and LeMANS NS simulations agree well with those from DSMC through much of the forebody shock layer, but considerable disagreement is found in the wake. The BGK-Euler simulation gives particularly inaccurate results throughout the flowfield, and reduced accuracy is also found for the BGK simulation in wake regions far from the axis. Discrepancies observed between BGK and BGK-NS results in the wake seem to indicate that, in the hybrid BGK-NS simulation, the NS domain may include areas of strong nonequilibrium within the wake where BGK calculations would be more appropriate. This assumption is reinforced by differences, discussed above, between Figs. 13 and 14. As expected, very good agreement is found between BGK, BGK-NS and DSMC simulations within the shock layer upstream of the cylinder.

Figures 16 and 17 show contours of normalized density and temperature, respectively, from simulations of the $\mathrm{Kn}=0.03$ case. Similar trends are found in both figures as in Fig. 15. As mentioned above in the discussion of Fig. 15, relatively good agreement is found in Figs. 16 and 17 between most simulation results around the shock layer and forebody boundary layer, while particularly large differences from DSMC are observed in results from the hybrid BGK-Euler simulation. The large error in BGK-Euler results is not particularly surprising, because - unlike BGK, DSMC, Boltzmann and NS calculations - the Euler calculations performed in continuum regions completely neglect effects of physical viscosity and diffusive transport, and any differences from a truly inviscid flow are due only to artificial viscosity.

In Fig. 18, the variation in normalized velocity along the stagnation streamline is plotted for all simulations of the $\mathrm{Kn}=0.03$ case. Very good agreement is found between DSMC and LeMANS NS results, whereas the UFS NS result shows a significantly thinner shock with a corresponding reduction of roughly $10 \%$ in the shock standoff distance. Differences between the two NS curves in Fig. 18 can be attributed primarily to the use of very different schemes. As mentioned above, the UFS NS solver uses a kinetic scheme of $\mathrm{Xu},{ }^{18}$ while LeMANS uses a modified form of Steger-Warming flux vector splitting. ${ }^{17}$ Although the scheme of $\mathrm{Xu}$ is expected to provide improved accuracy relative to the Steger-Warming method through a reduction in diffusive and dissipative effects, the better agreement with DSMC found in the LeMANS results seems to indicate that, for this case, the Xu scheme implementation in UFS may not allow sufficient diffusive transport. Further investigation is required to assess whether discrepancies between UFS NS and DSMC results in Fig. 18 are due to numerical problems or physical approximations in the UFS NS solver.

Nearly identical curves are shown in Fig. 18 from the BGK and hybrid BGK-NS simulations, although both give relatively poor agreement with DSMC toward the upstream portion of the shock. A comparison of BGK and DSMC results in this region indicates that the BGK approximation of the Boltzmann equation may produce excessive diffusive transport within the shock. As stated above in the discussion of Figs. 6 and 8, this same trend (excess diffusion in the shock) is also found in a comparison of BGK, Boltzmann and DSMC results for the $\mathrm{Kn}=0.3$ case. In Fig. 18, as in Figs. 15 through 17, the greatest differences from DSMC are observed in results from the hybrid BGK-Euler simulation. In relation to DSMC, the BGK-Euler simulation overestimates the shock standoff distance by approximately $12 \%$. Despite significant discrepancies in the location and thickness of the shock, all six curves shown in Fig. 18 give relatively good agreement within the post-shock and boundary layer regions.

Figures 19 and 20 show contours of normalized density and temperature, respectively, for the $\mathrm{Kn}=0.03$ case. Similar trends are found in both figures as in Fig. 18, with comparable differences in shock thickness and shock standoff distance based on either density or temperature. The only prominent trends in Figs. 19 and 20 which are not apparent in Fig. 18 relate to boundary layer thickness. The BGK, BGK-NS and BGK-Euler simulations all noticeably overestimate the boundary layer thickness, as measured by density or temperature, relative to DSMC. Corresponding gradients within the boundary layer are lower in these UFS simulations than in DSMC. In contrast, only very small differences in boundary layer thickness are found in Figs. 19 and 20 between results from UFS NS, LeMANS NS and DSMC simulations. Differences in boundary layer thickness are likely due to excess diffusive transport in BGK calculations, which is also thought to cause the overly thick shocks observed in Figs. 6, 8 and 18.

The surface pressure coefficient for $\mathrm{Kn}=0.03$ simulations is plotted in Fig. 21. Note that values from the UFS NS simulation are not provided, because the version of UFS used in this study did not allow output of surface quantities at wall boundary cells within the NS domain. Good agreement is observed along the forebody between all pressure coefficient curves, while quantitative (but not qualitative) differences are shown for $\theta>90^{\circ}$. The greatest disagreement with DSMC pressure coefficient values at $\theta>90^{\circ}$ is found in results from the BGK-Euler and NS simulations. As discussed above, error in NS results is likely due to effects of continuum breakdown, while BGKEuler inaccuracies can be mainly attributed to a lack of diffusive transport in calculations for the Euler equations. Although BGK-NS and NS results are in good agreement for $\theta>150^{\circ}$, neither compares well over this portion of the surface with either BGK or DSMC. This trend seems to indicate that, as mentioned in the discussion of Fig. 15, 
the NS domain in the BGK-NS simulation may include rarefied wake regions which should be assigned to the BGK domain.

Figure 22 shows the variation in the surface friction coefficient for simulations of the $\mathrm{Kn}=0.03$ case. The most noticeable errors are found in the NS curve, which agrees reasonably well with DSMC over the forebody surface $(\theta$ $<90^{\circ}$ ) but is up to a factor of five greater than DSMC along the afterbody. Most of this discrepancy can be attributed to the lack of a velocity slip/temperature jump boundary condition in NS calculations; due to the high degree of continuum breakdown along the cylinder surface shown in Fig. 13, significant velocity slip is expected. Both DSMC and BGK calculations allow for velocity slip along the surface, and as expected, the lack of wall slip in NS calculations is associated with higher friction coefficient values in the NS results. All UFS simulations tend to slightly over predict surface shear stress along the forebody, with an overestimate relative to DSMC of up to around $50 \%$ in the BGK results. Note the slope discontinuity in all curves around $\theta=165^{\circ}$, which corresponds to boundary layer separation at the edge of a recirculation zone. In comparing the location of this discontinuity between results from different simulations, we find good overall agreement in the size of the recirculating region. Relative to DSMC, the maximum difference in the $\theta$ range for the recirculating region is found in results from the BGK-NS simulation, which overestimates this range by about $30 \%$.

In Fig. 23, the surface heat transfer coefficient is plotted for simulations at $\mathrm{Kn}=0.03$. As with the friction coefficient values shown in Fig. 22, the NS simulation slightly over predicts the heat transfer over much of the forebody surface, and greatly over predicts this quantity along the afterbody. A maximum relative error (with respect to the DSMC value) of approximately $32 \%$ is found at $\theta=148^{\circ}$. Significant discrepancies are observed between DSMC and the various UFS results, with a roughly $20 \%$ underestimate in heat transfer over much of the surface from the BGK-NS simulation and a somewhat smaller overestimate from the BGK simulation. As mentioned in the discussion of Fig. 11, all UFS simulations exhibit an unphysical "stair step" pattern in the $\theta$ range between $90^{\circ}$ and $150^{\circ}$. These errors, as well as a lack of smoothness in surface heat transfer curves at smaller $\theta$ values, are likely caused by problems with gradient evaluations involving Cartesian cut cells. Note that UFS BGK and NS calculations are entirely deterministic, and fluctuations observed in the UFS results are not due to the type of statistical scatter found in DSMC.

\section{Comparison of simulation results for $\mathrm{Kn}=\mathbf{0 . 0 0 3}$}

Figure 24 shows the computational meshes used in UFS NS and LeMANS NS simulations at $\mathrm{Kn}=0.003$. While the UFS mesh is able to capture high gradient regions within areas of increased refinement, no automatic grid adaption is possible using LeMANS. The LeMANS mesh, which was manually generated using the commercial GAMBIT code, includes a structured region surrounding the cylinder, and an unstructured region of uniform-sized triangular cells which covers much of the wake. In creating the LeMANS mesh, particular effort was made to ensure shock alignment and grid independence. As described in section III, stretched (i.e. high aspect ratio) cells are used in the boundary layer to avoid unnecessarily small cell dimensions in the surface-tangent direction.

In Fig. 25, contours are shown for the maximum gradient length local Knudsen number, as computed from the LeMANS simulation at $\mathrm{Kn}=0.003$. As expected for such a small $\mathrm{Kn}$ value, continuum breakdown (where $\mathrm{Kn}_{\mathrm{GLL}-\max }$ $>0.05$ ) is found to occur only in narrow regions around the bow shock and forebody boundary layer, as well as in portions of the nearfield wake region. This indicates that the NS equations are valid over nearly the entire flowfield, and relatively small errors should result from the near-equilibrium assumptions underlying these equations.

In Fig. 26, contours of normalized bulk velocity magnitude are compared from the two NS simulations at Kn $=$ 0.003. Very good overall agreement is found between the two sets of contour lines, with the largest discrepancies observed in the farfield wake region. Differences in the shape of the $\mathrm{V} / \mathrm{V}_{\infty}=0.8$ contour line far from the axis may be attributed to the presence of a UFS symmetry boundary condition at $\mathrm{y}=1 \mathrm{~m}$. Particularly good agreement is found in the shock layer, with a shock standoff distance based on velocity contours which is approximately $5 \%$ larger in the LeMANS simulation than in the UFS simulation. Figures 27 and 28 show contours of normalized density and temperature, respectively, from the $\mathrm{Kn}=0.003$ simulations. In both figures, similar levels of disagreement are found as in Fig. 20, with particularly large differences in the post-shock region far from the axis and in the wake.

The variation in bulk velocity, density and temperature along the stagnation streamline is plotted in Figs. 29, 30 and 31 from the NS simulations at $\mathrm{Kn}=0.003$. All three figures show very similar trends, with excellent agreement in post-shock values and boundary layer profiles, along with a thicker shock and slightly larger shock standoff distance in the LeMANS simulation. Based on the location of maximum gradients, the UFS simulation is found to underestimate the shock standoff distance by between $4 \%$ and $5 \%$ relative to the LeMANS simulation. 


\section{Drag coefficient comparison}

In Fig. 32, drag coefficients $C_{d}$ calculated from UFS, DSMC and NS simulations are plotted as a function of Kn. Data from experiments ${ }^{21}$ and additional simulations are also included in the figure for comparison. Symbols labeled "Tratio $=1.2$ " indicate that the ratio of cylinder wall temperature to freestream temperature is 1.2 , as is the case for all simulations described above and included in Fig. 1. Likewise, symbols labeled "Tratio $=4$ " indicate simulations for which the cylinder wall temperature is four times that of the freestream gas. Several additional simulations using this higher wall temperature have been performed over a wide range of Kn values. Among these added simulations, five are run using the LeMANS NS solver, seven use MONACO, and seven employ the BGK module in UFS. Additional data points, labeled "Boltzmann*, Tratio $=4$ " are taken from published results of UFS Boltzmann simulations by Kolobov et al. ${ }^{9}$

In Fig. 32, the cylinder surface temperature is shown to have little if any noticeable impact on $\mathrm{C}_{\mathrm{d}}$ over the full Kn range considered. Generally good agreement is found between UFS Boltzmann, DSMC and measured values even around the maximum Kn of 10. Good agreement is also found between all UFS, DSMC, NS and experimental data for $\mathrm{Kn}<0.1$. At higher $\mathrm{Kn}$, however, large overestimates relative to the experimental values are observed in NS $\mathrm{C}_{\mathrm{d}}$ predictions, with an overestimate of roughly $70 \%$ at $\mathrm{Kn}=1$. Smaller but still significant errors in $\mathrm{C}_{\mathrm{d}}$ are shown in BGK results for $\mathrm{Kn}>0.3$, with an underestimate relative to $\mathrm{DSMC}$ of about $7 \%$ at $\mathrm{Kn}=10$. A noticeable increase with $\mathrm{Kn}$ is found in the level of disagreement between DSMC and BGK results. As no such Kn-dependent discrepancies are found between DSMC and Boltzmann results, this disagreement may be attributed to inaccuracies in the BGK collision integral approximation which become increasingly influential at higher Kn.

\section{Conclusions}

Results have been presented from a series of simulations for hard sphere monatomic gas flows over a cylinder at a freestream Mach number of 4 . A wide range of global Knudsen numbers have been considered, including one case $(\mathrm{Kn}=0.3)$ involving strong nonequilibrium over nearly the entire flowfield, a second case $(\mathrm{Kn}=0.03)$ involving significant regions of both continuum and rarefied flow, and a third case $(\mathrm{Kn}=0.003)$ for which the NS equations are valid over nearly the full simulation domain. These flow problems have been used to evaluate various models and combinations of models in the UFS code. This code holds great promise in simulating a range of hypersonic gas flow problems, particularly those involving strong two-way coupling between rarefied and continuum regions, as well as unsteady multiscale flows and other flows with translational nonequilibrium for which the inherent statistical scatter in DSMC is unacceptable or problematic.

In comparing efficiency and accuracy of UFS calculations relative to MONACO DSMC and LeMANS NS simulations, a variety of attributes and areas of potential improvement in UFS have been identified. First, the ease with which multiple methods, intended for different Kn regimes, can be strongly coupled within a single simulation is an important positive characteristic for UFS, and capabilities for automatic tree-based Cartesian mesh adaptation greatly reduce the simulation setup time and simplify setup procedures for the user. Unlike DSMC or hybrid DSMCCFD techniques, UFS has no problems or complications associated with statistical scatter, and there is no loss in precision if UFS calculations are immediately stopped once steady state conditions have been reached. Future code development for UFS may include inclusion of internal excitation or chemistry models which rely heavily on the velocity distribution function, and which may be more accurate when used in direct numerical solutions to the Boltzmann equation than in DSMC.

The major drawback in UFS, as demonstrated in this study, is the enormous computational expense of the Boltzmann solver in relation to DSMC. The Boltzmann simulation of the $\mathrm{Kn}=0.3$ case was over two orders of magnitude more expensive than the corresponding DSMC simulation. While computational expense may not be a driving concern for this relatively simple type of problem given the desired levels of simulation accuracy and precision, the large discrepancy in simulation efficiency implies that for a given set of computational resources, a DSMC code should be capable of simulating certain more complex rarefied gas flows which would be prohibitively expensive to simulate using the Boltzmann solver in UFS. However, it should be emphasized that - as discussed above - direct numerical simulation of the Boltzmann equation has several advantages over DSMC which may outweigh any disadvantages in efficiency.

Large efficiency gains were realized when BGK calculations were used in place of Boltzmann calculations, although BGK simulations were found to give reduced accuracy relative to Boltzmann and DSMC simulations. Much of the error in BGK results is presumably due to underlying approximations in the BGK equation. Additional errors were found in surface quantities computed using either BGK or Boltzmann solvers; these errors are most likely associated with gradient approximations involving cut cell boundaries, and should be reduced or eliminated through the use of an immersed boundary method. 
To improve efficiency in the UFS Boltzmann solver without sacrificing simulation accuracy, several possible approaches are suggested: First, a reduced number of nodes in velocity space could potentially be used. This may be accomplished by means of velocity grid adaptation, where calculations for each cell in physical space would involve only those points in velocity space with a relatively high probability density. A related approach would allow for optimized non-uniform grids in velocity space, where the velocity space nodes would be selected in a manner similar to that used in Gaussian quadrature. As described in Ref. 9, the NtCN method currently employed in UFS to evaluate the Boltzmann collision integral should allow for either option.

Acceptable accuracy may be found using fewer velocity space nodes, even without velocity grid adaptation, if only the lower moments of the velocity distribution are needed to calculate macroscopic output quantities of interest. ${ }^{22}$ However, a more refined velocity grid is likely required in simulations involving higher Mach numbers or stronger translational nonequilibrium, or in simulations for which higher velocity distribution moments are used to model physical phenomena such as internal energy excitation or chemistry.

Comparable accuracy could also be achieved with a coarser velocity grid if the Boltzmann solver is modified for higher order accuracy in velocity space, although the required modifications may be prohibitively complicated and involve considerable method and algorithm development. Another possible approach to increase efficiency would involve minimizing, for a desired level of accuracy, the number of summation terms used to approximate the collision integral. Related efficiency gains could potentially be found by testing alternate low discrepancy sequences to select velocity space grid points used in this summation.

\section{Acknowledgments}

The authors gratefully acknowledge the Michigan/AFRL/Boeing Collaborative Center in Aeronautical Sciences, which provides funding for the work described in this paper. The authors would also like to thank Robert Arslanbekov and Vladimir Kolobov for several helpful discussions and insightful suggestions over the course of this work.

\section{References}

${ }^{1}$ Bird, G. A., Molecular Gas Dynamics and the Direct Simulation of Gas Flows, Clarendon Press, Oxford, 1994.

${ }^{2}$ Vincenti, W. G., and Kruger, C. H., Introduction to Physical Gas Dynamics, Krieger Publishing, Malabar, Florida, 1986.

${ }^{3}$ Schwartzentruber, T. E., and Boyd, I. D., "A Modular Particle-Continuum Numerical Method for Hypersonic Nonequilibrium Gas Flows," Journal of Computational Physics, Vol. 225, 2007, pp. 1159-1174.

${ }^{4}$ Wu, J.-S., Lian, Y.-Y., Cheng, G., Koomullil, R. P., and Tseng, K.-C., "Development and Verification of a Coupled DSMCNS Scheme Using Unstructured Mesh,” Journal of Computational Physics, Vol. 219, 2006, pp. 579-607.

${ }^{5}$ Kaplan, C. R., Liu, J., and Oran, E. S., "Parallel Hybrid Method for Subsonic Flows: Coupling and Load-Balancing Challenges," AIAA Paper 2006-992, 2006.

${ }^{6}$ Garcia, A. L., Bell, J. B., Crutchfield, W. Y., and Alder, B. J., "Adaptive Mesh and Algorithm Refinement Using Direct Simulation Monte Carlo," Journal of Computational Physics, Vol. 154, 1999, pp. 134-155.

${ }^{7}$ Wadsworth, D. C., and Erwin, D. A., "Two-Dimensional Hybrid Continuum/Particle Approach for Rarefied Flows," AIAA Paper 92-2975, 1992.

${ }^{8}$ Hash, D. B., and Hassan, H. A., "Assessment of Schemes for Coupling Monte Carlo and Navier-Stokes Solution Methods," Journal of Thermophysics and Heat Transfer, Vol. 10, No. 2, 1996, pp. 242-249.

${ }^{9}$ Kolobov, V. I., Arslanbekov, R. R., Aristov, V. V., Frolova, A. A., and Zabelok, S. A., "Unified Solver for Rarefied and Continuum Flows with Adaptive Mesh and Algorithm Refinement," Journal of Computational Physics, Vol. 223, 2007, pp. 589608 .

${ }^{10}$ Kolobov, V. I., Bayyuk, S. A., Arslanbekov, R. R., Aristov, V. V., Frolova, A. A., and Zabelok, S. A., "Construction of a Unified Continuum/Kinetic Solver for Aerodynamic Problems," Journal of Spacecraft and Rockets, Vol. 42, No. 4, 2005, pp. 598-606.

${ }^{11}$ Josyula, E., Arslanbekov, R. R., Kolobov, V. I., and Gimelshein, S. F., "Evaluation of Kinetic/Continuum Solver for Hypersonic Nozzle-Plume Flow," Journal of Spacecraft and Rockets, Vol. 45, No. 4, 2008, pp. 665-676.

${ }^{12}$ Arslanbekov, R. R., Kolobov, V. I., Frolova, A., Zabelok, S., and Josyula, E., "Evaluation of a Unified Kinetic/Continuum Solver for Computing Heat Flux in Hypersonic Blunt Body Flows," AIAA Paper 2007-4544, 2007.

${ }^{13}$ Dinavahi, S., and Josyula, E., "Simulation of Mach 3 Cylinder Flow using Kinetic and Continuum Solvers," Department of Defense HPCMP User Group Conference, San Diego, CA, 2009.

${ }^{14}$ Popinet, S., "Gerris: A Tree-Based Adaptive Solver for the Incompressible Euler Equations in Complex Geometries," Journal of Computational Physics, Vol. 190, 2003, pp. 572-600.

${ }^{15}$ Dietrich, S., and Boyd, I. D., "Scalar and Parallel Optimized Implementation of the Direct Simulation Monte Carlo Method," Journal of Computational Physics, Vol. 126, 1996, pp. 328-342.

${ }^{16}$ Scalabrin, L. C., and Boyd, I. D., "Development of an Unstructured Navier-Stokes Solver for Hypersonic Nonequilibrium Aerothermodynamics," AIAA Paper 2005-5203, 2005. 


\footnotetext{
${ }^{17}$ Laney, C. B., Computational Gasdynamics, Cambridge University Press, 1998.

${ }^{18} \mathrm{Xu}, \mathrm{K}$., "A Gas-Kinetic BGK Scheme for the Navier-Stokes Equations and its Connection with Artificial Dissipation and Godunov Method,” Journal of Computational Physics, Vol. 171, 2001, pp. 289-335.

${ }^{19}$ Wang, W., and Boyd, I. D., "Predicting Continuum Breakdown in Hypersonic Viscous Flows," Physics of Fluids, Vol. 15, No. 1, 2003, pp. 91-100.

${ }^{20}$ Lofthouse, A. J., "Hypersonic Blunt Body Thermophysics Using a Unified Kinetic/Continuum Solver," AIAA Paper 20093838, 2009.

${ }^{21}$ Maslach, G. J., and Schaaf, S. A., "Cylinder Drag in the Transition from Continuum to Free-Molecule Flow," Physics of Fluids, Vol. 6, No. 3, 1963, pp. 315-321.

${ }^{22}$ Josyula, E., Xu, K., Suchyta, C. J., and Bailey, W. F., "Kinetic Methods for Solving the Internal Structure of Shock Waves," AIAA Paper 2009-3841, 2009.
}

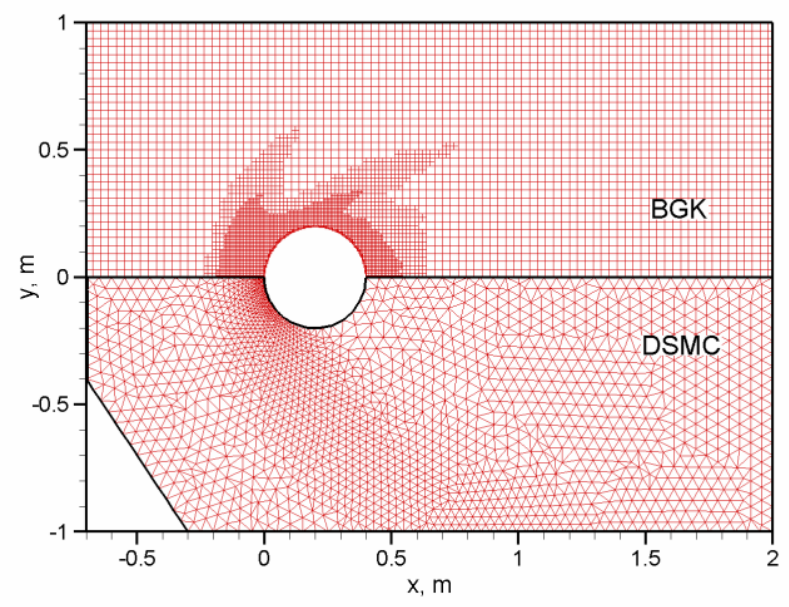

Figure 1. Meshes for UFS BGK and DSMC simulations at $\mathrm{Kn}=0.3$.

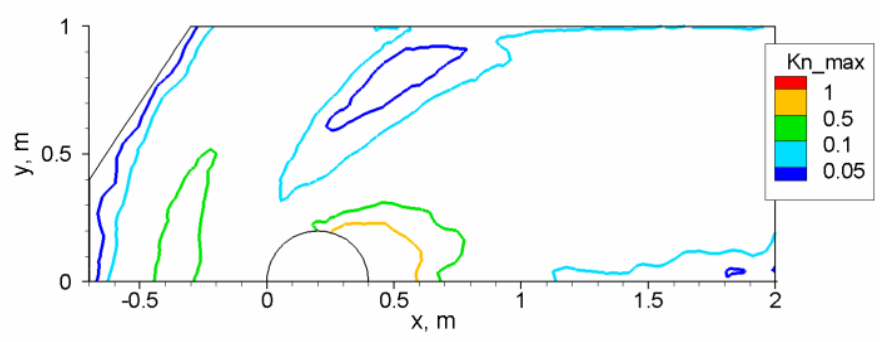

Figure 2. Contours of the maximum gradient length local Knudsen number from the DSMC simulation at $\mathrm{Kn}=\mathbf{0 . 3}$. 


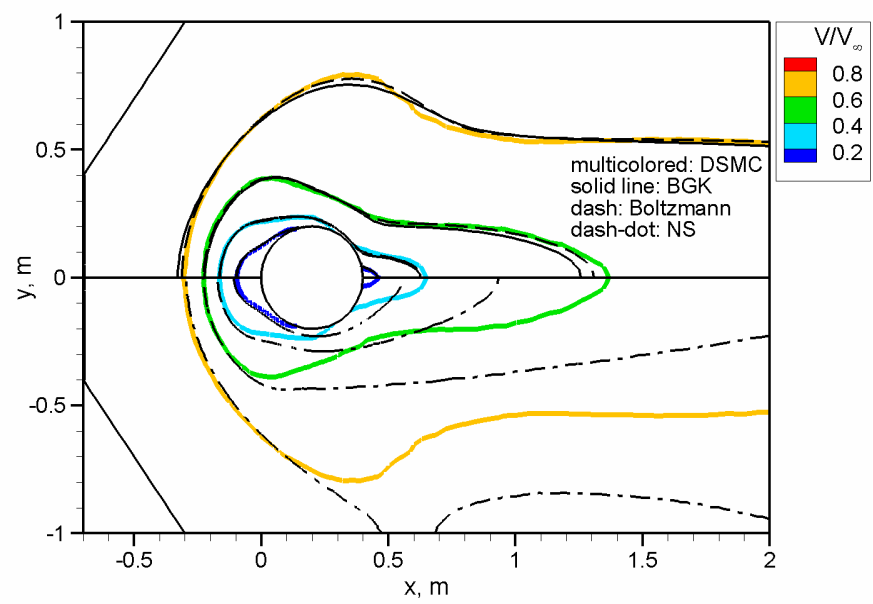

Figure 3. Contours of bulk velocity magnitude for $\mathrm{Kn}=0.3$. BGK and Boltzmann simulations are both run on UFS, the NS simulation is performed using LeMANS, and the DSMC simulation is run on MONACO.

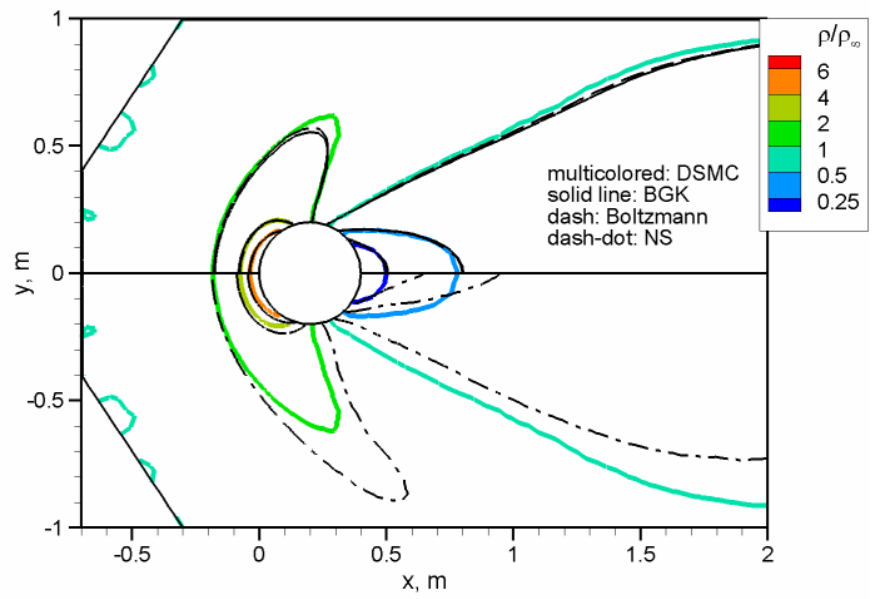

Figure 4. Contours of density for $\mathrm{Kn}=\mathbf{0 . 3}$. UFS is used for BGK and Boltzmann simulations.

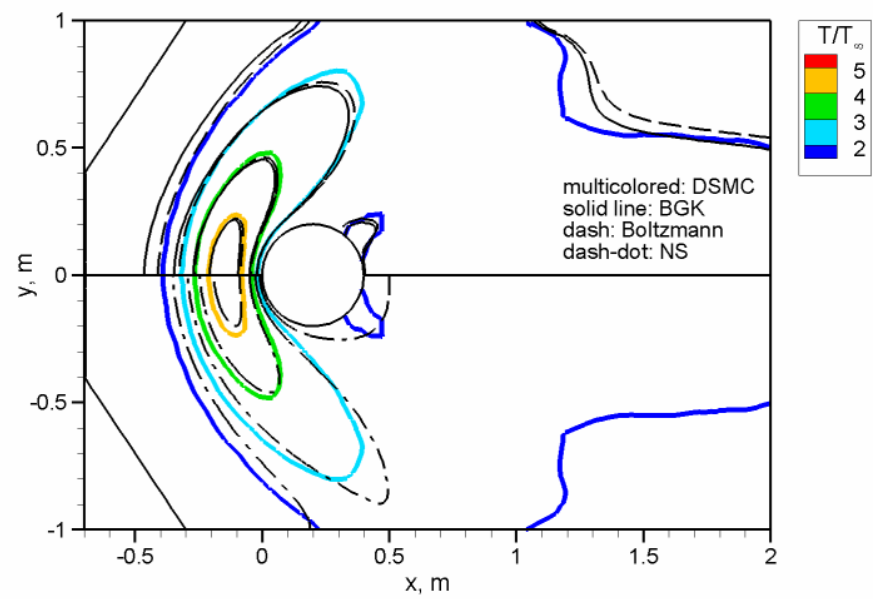

Figure 5. Contours of temperature for $\mathrm{Kn}=0.3$. UFS is used for BGK and Boltzmann simulations. 


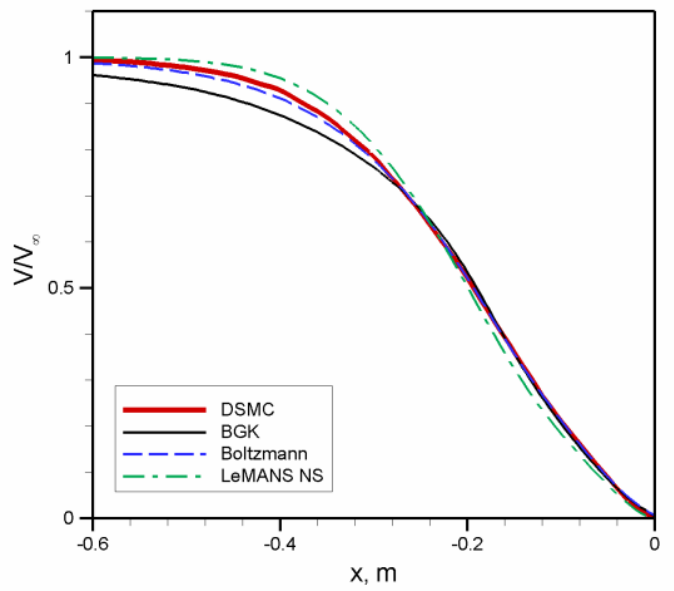

Figure 6. Bulk velocity along the stagnation streamline for $\mathrm{Kn}=0.3$. UFS is used for BGK and Boltzmann simulations.

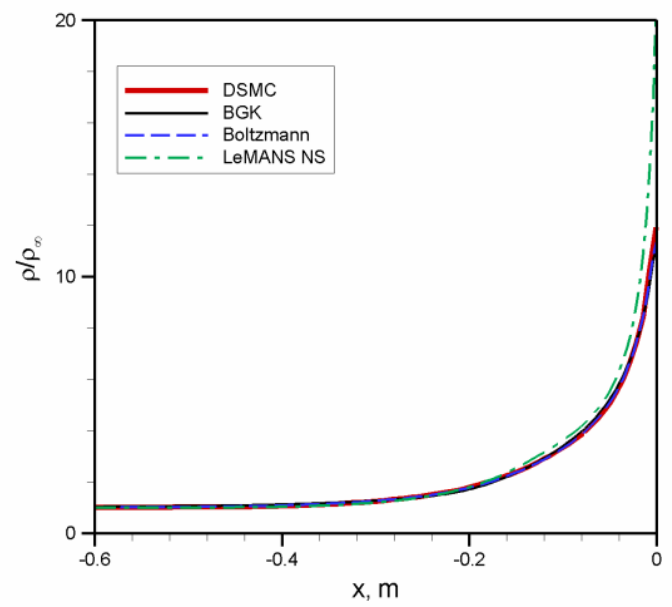

Figure 7. Density along the stagnation streamline for $\mathrm{Kn}=0.3$. UFS is used for BGK and Boltzmann simulations.

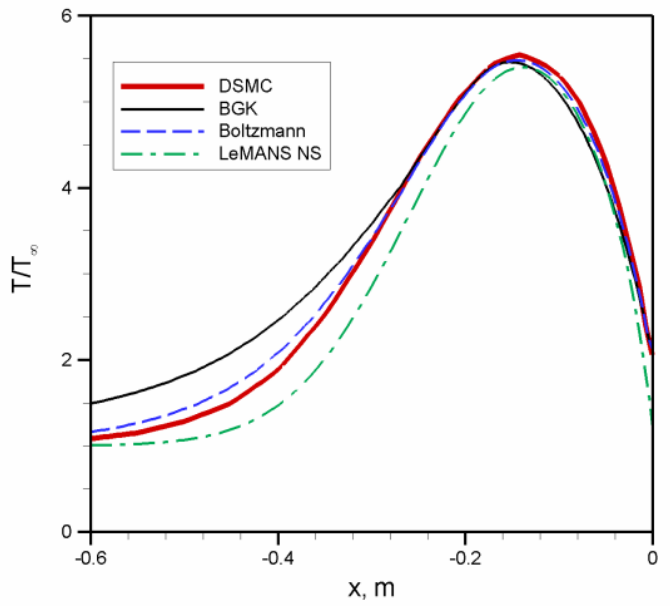

Figure 8. Temperature along the stagnation streamline for $\mathrm{Kn}=0.3$. UFS is used for BGK and Boltzmann simulations. 


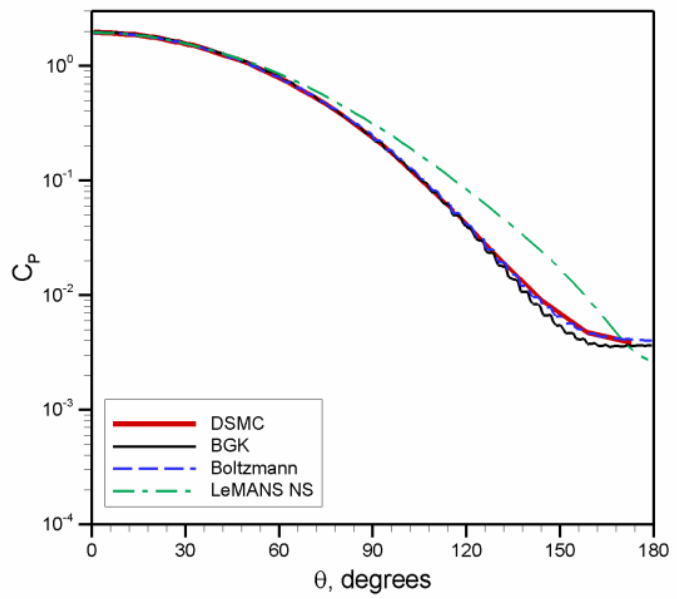

Figure 9. Surface pressure coefficient for Kn = 0.3. UFS is used for BGK and Boltzmann simulations.

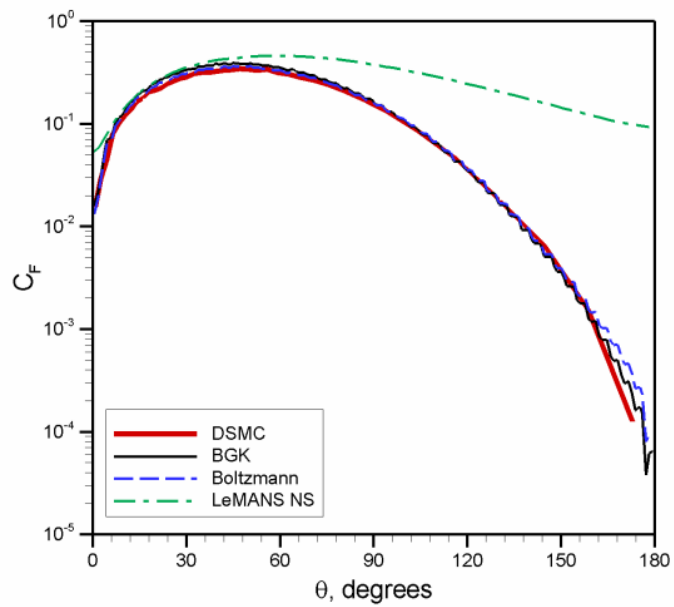

Figure 10. Surface friction coefficient for $\mathrm{Kn}=0.3$. UFS is used for BGK and Boltzmann simulations.

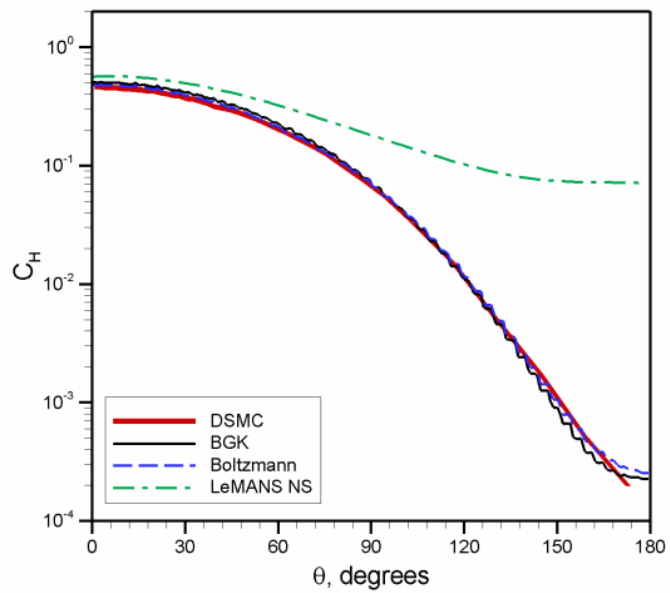

Figure 11. Surface heat transfer coefficient for $\mathrm{Kn}=0.3$. UFS is used for BGK and Boltzmann simulations. 


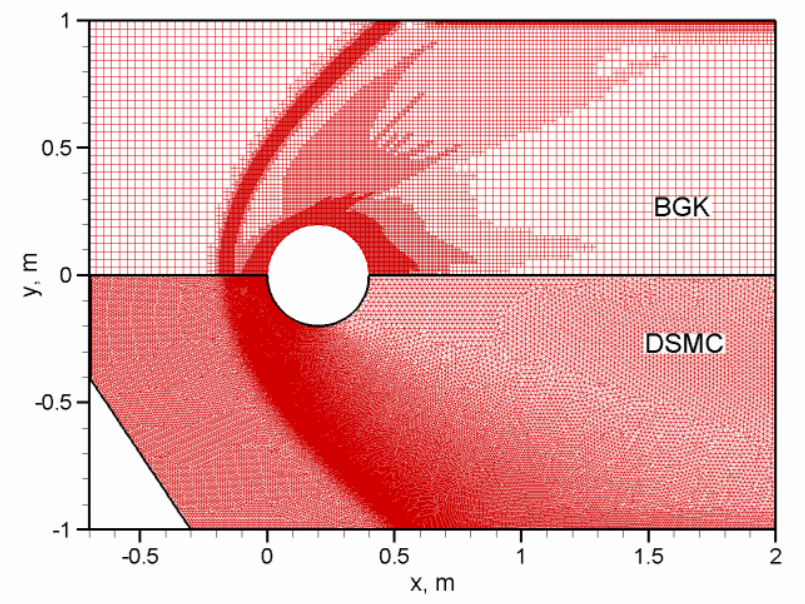

Figure 12. Meshes for UFS BGK and MONACO DSMC simulations at $\mathrm{Kn}=0.03$.

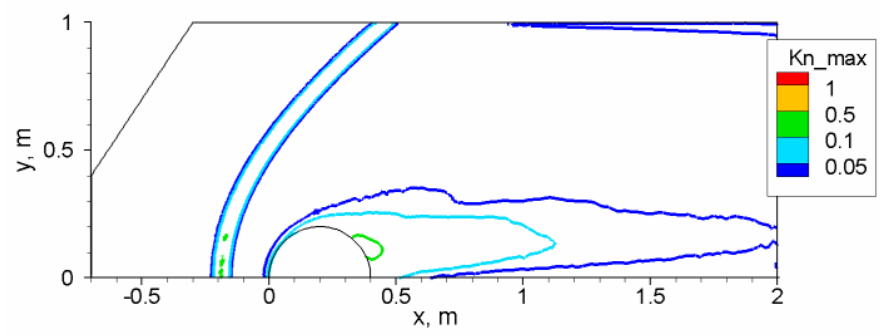

Figure 13. Contours of the maximum gradient length local Knudsen number from the DSMC simulation at Kn $=0.03$.

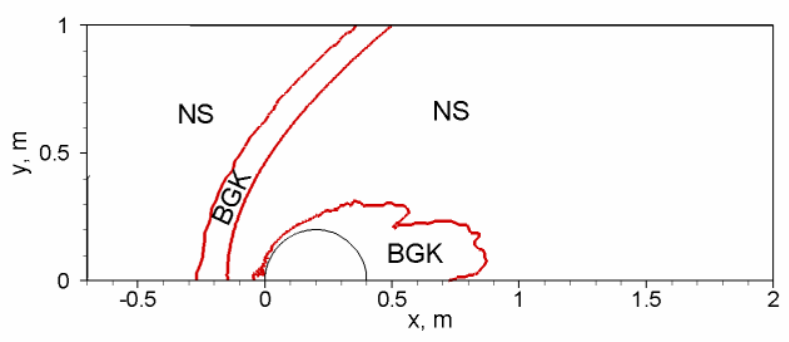

Figure 14. Continuum and rarefied domains for the UFS BGK-NS simulation at $\mathrm{Kn}=0.03$. 


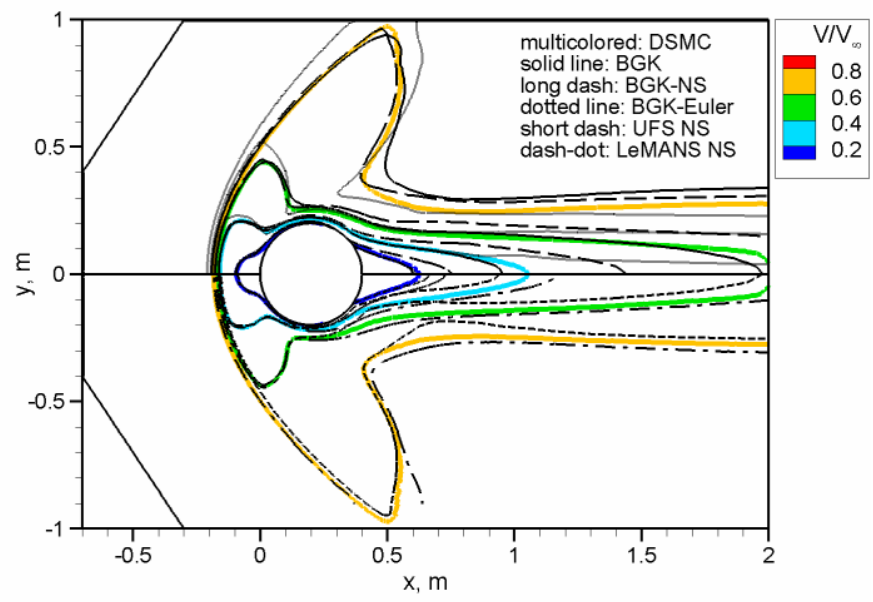

Figure 15. Contours of bulk velocity magnitude for $\mathrm{Kn}=\mathbf{0 . 0 3}$. UFS is used for all simulations except the LeMANS NS simulation and the MONACO DSMC simulation.

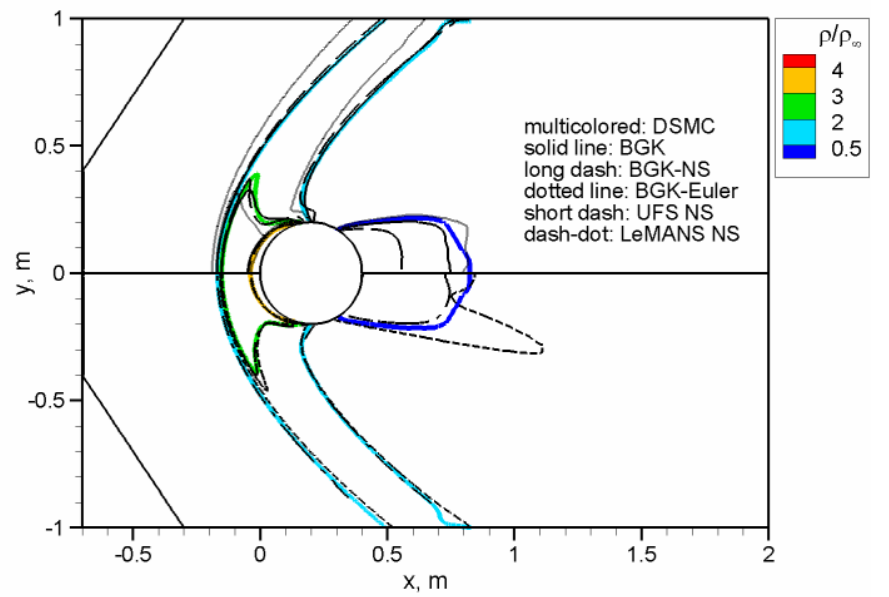

Figure 16. Contours of density for $\mathrm{Kn}=\mathbf{0 . 0 3}$. UFS is used for BGK, BGK-NS, and BGK-Euler simulations.

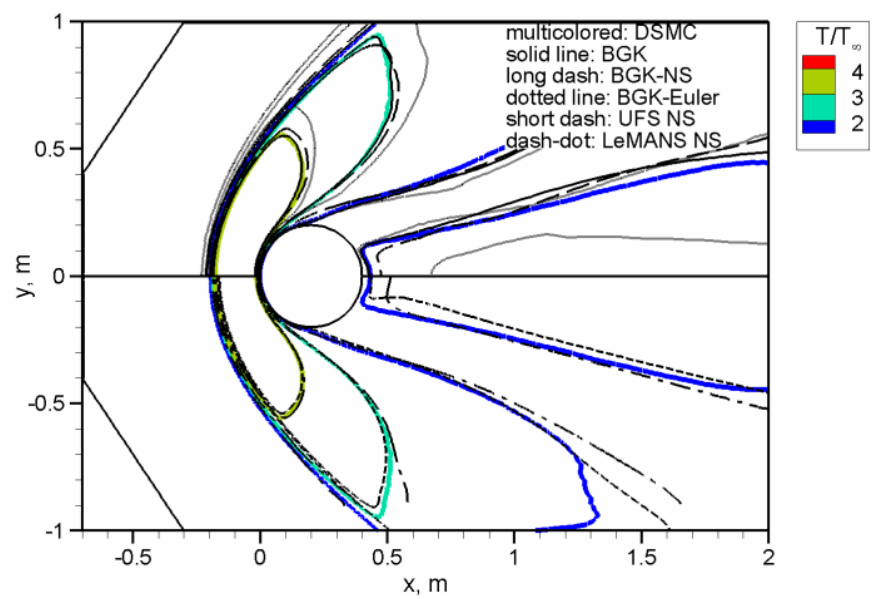

Figure 17. Contours of density for Kn $=0.03$. UFS is used for BGK, BGK-NS, and BGK-Euler simulations. 


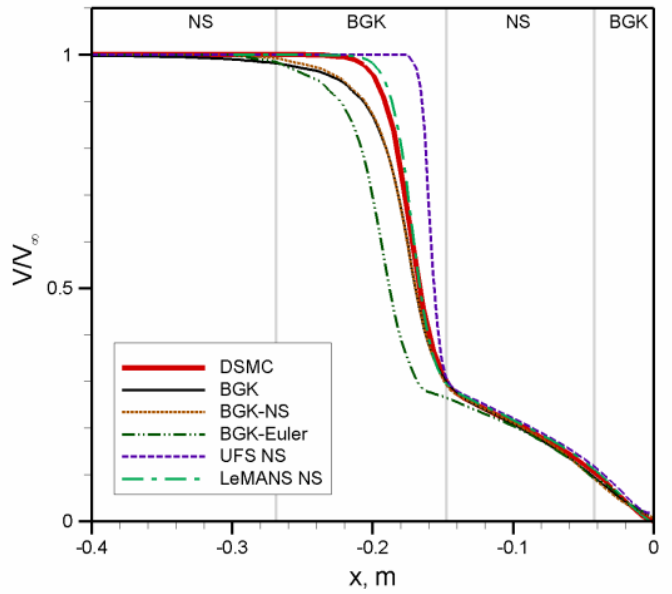

Figure 18. Bulk velocity along the stagnation streamline for $\mathrm{Kn}=0.03$. UFS is used for BGK, BGK-NS, and BGK-Euler simulations.

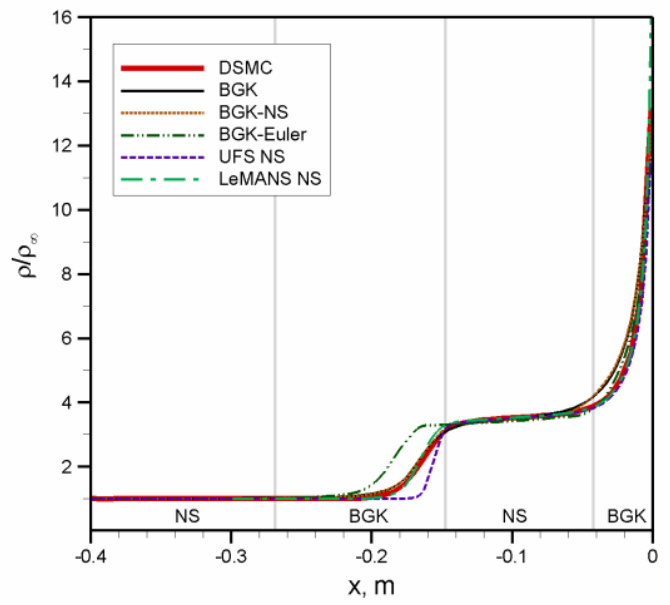

Figure 19. Density along the stagnation streamline for $\mathrm{Kn}=0.03$. UFS is used for BGK, BGK-NS, and BGK-Euler simulations.

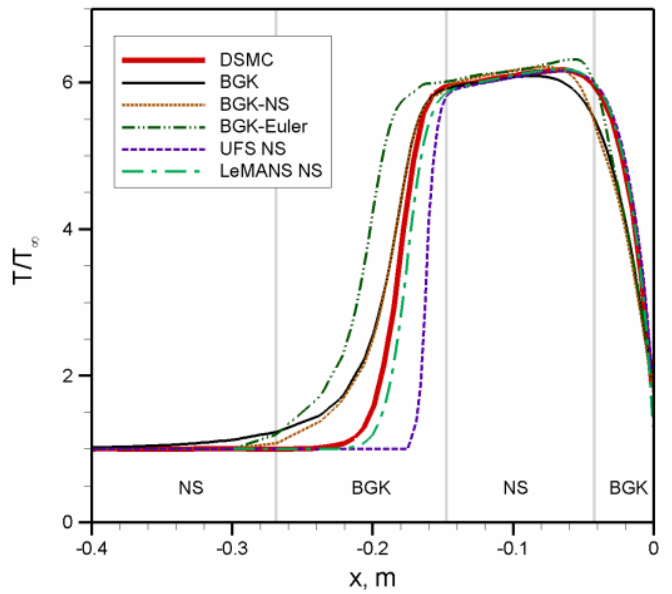

Figure 20. Temperature along the stagnation streamline for $\mathrm{Kn}=\mathbf{0 . 0 3}$. UFS is used for BGK, BGK-NS, and BGK-Euler simulations. 


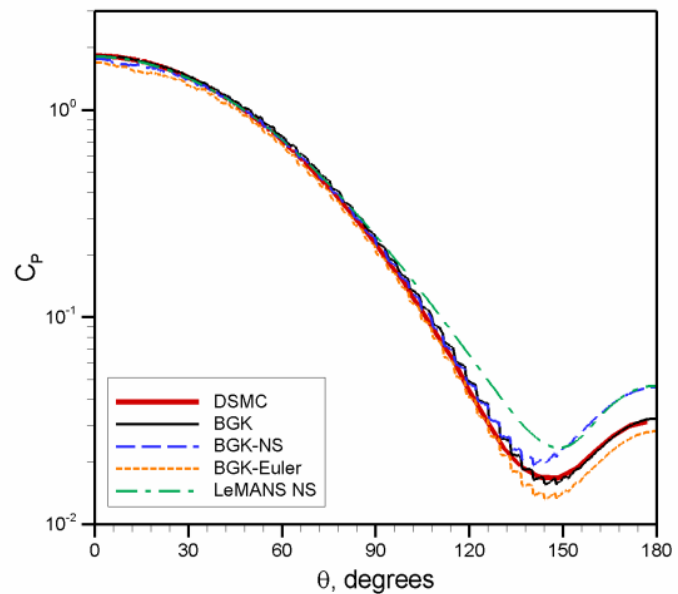

Figure 21. Surface pressure coefficient for $\mathrm{Kn}=\mathbf{0 . 0 3}$. UFS is used for BGK, BGK-NS, and BGK-Euler simulations.

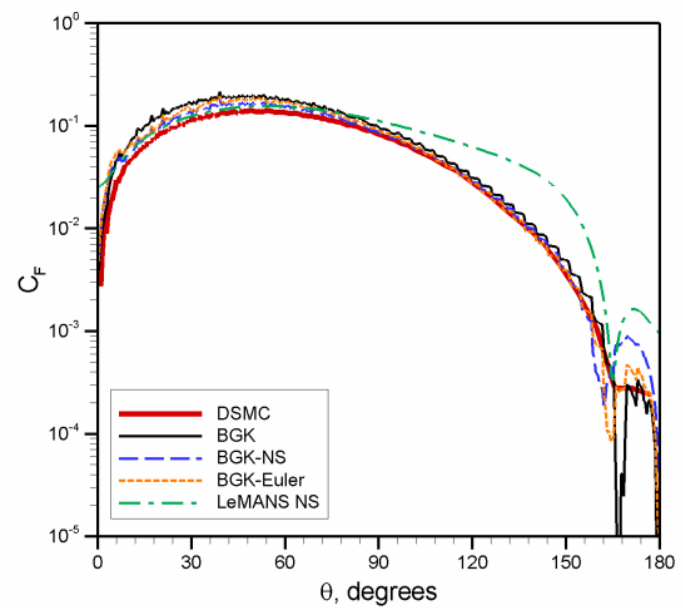

Figure 22. Surface friction coefficient for $\mathrm{Kn}=\mathbf{0 . 0 3}$. UFS is used for BGK, BGK-NS, and BGK-Euler simulations.

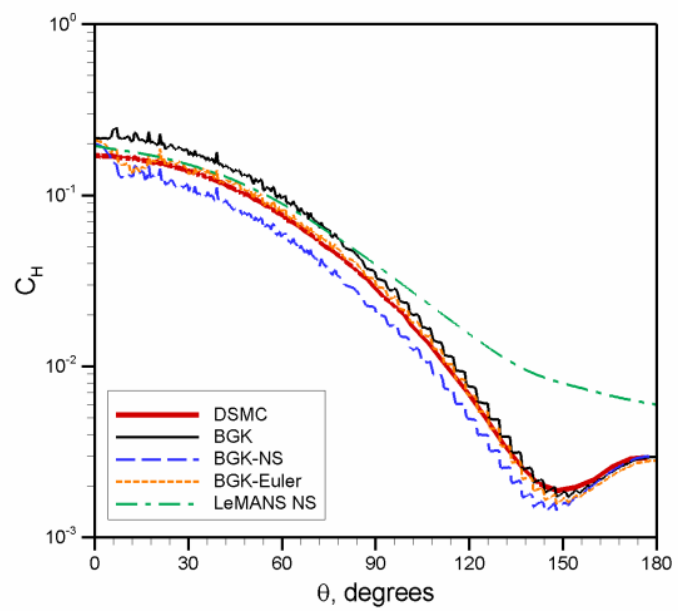

Figure 23. Surface heat transfer coefficient for $\mathrm{Kn}=\mathbf{0 . 0 3}$. UFS is used for BGK, BGK-NS, and BGK-Euler simulations. 


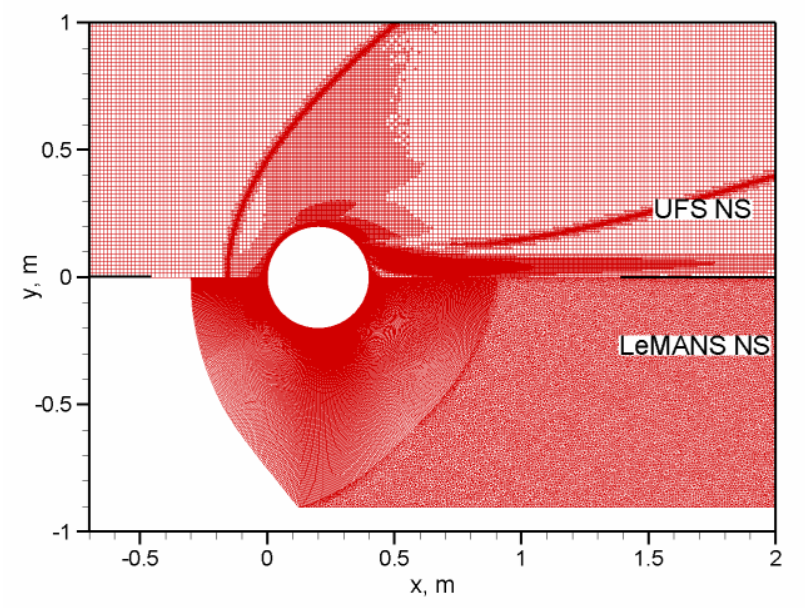

Figure 24. Meshes for UFS NS and LeMANS NS simulations at $\mathrm{Kn}=0.003$.

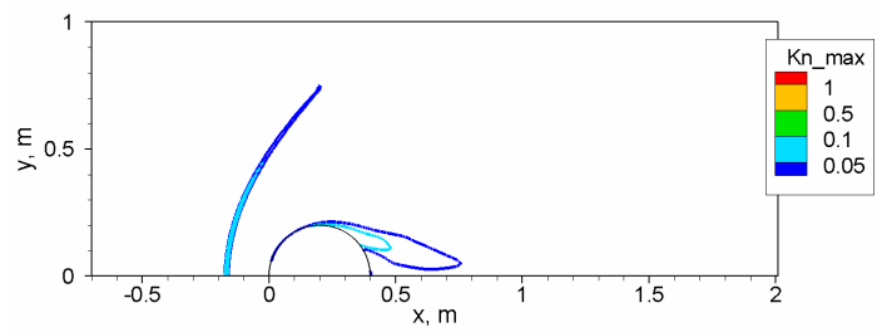

Figure 25. Contours of the maximum gradient length local Knudsen number from the LeMANS simulation at $\mathrm{Kn}=\mathbf{0 . 0 0 3}$.

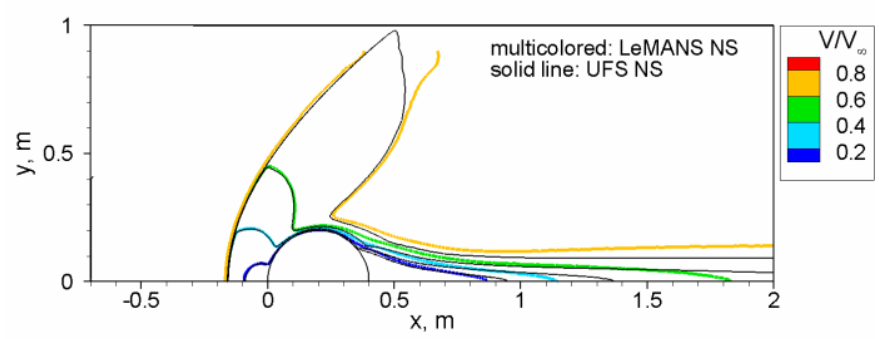

Figure 26. Contours of bulk velocity magnitude for $K n=0.003$.

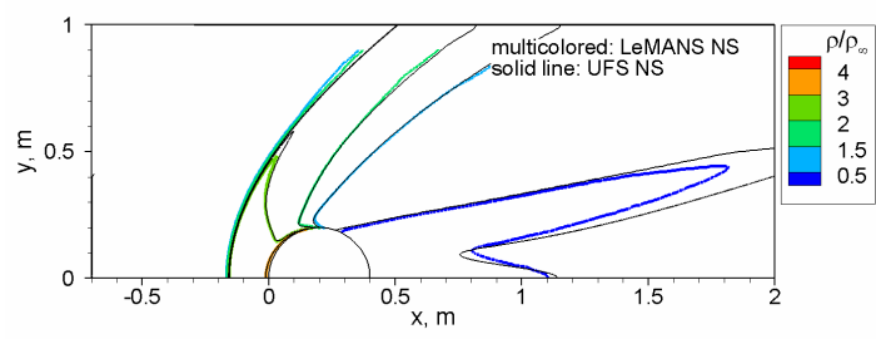

Figure 27. Contours of density for $\mathrm{Kn}=\mathbf{0 . 0 0 3}$. 


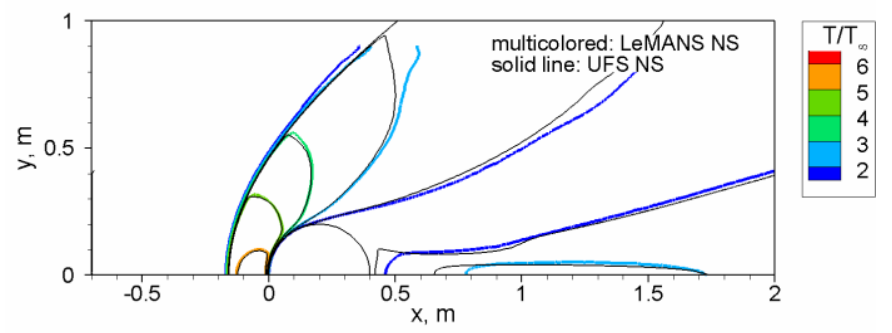

Figure 28. Contours of temperature for $\mathrm{Kn}=\mathbf{0 . 0 0 3}$.

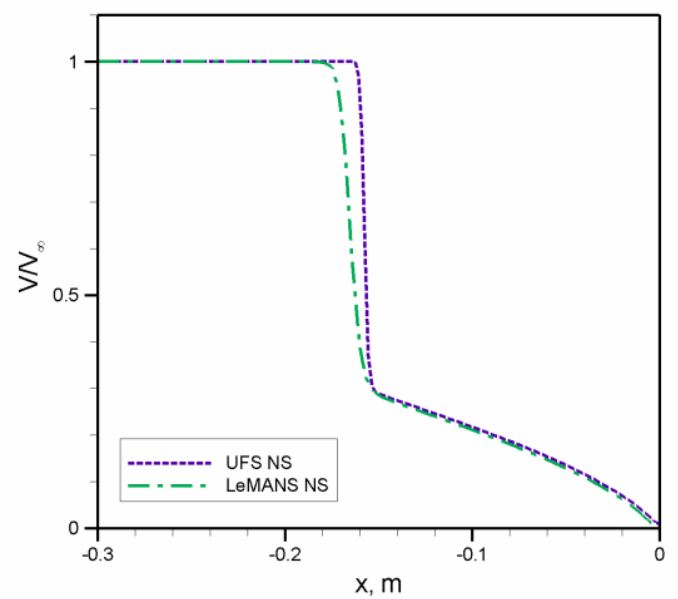

Figure 29. Bulk velocity along the stagnation streamline for $\mathrm{Kn}=\mathbf{0 . 0 0 3}$.

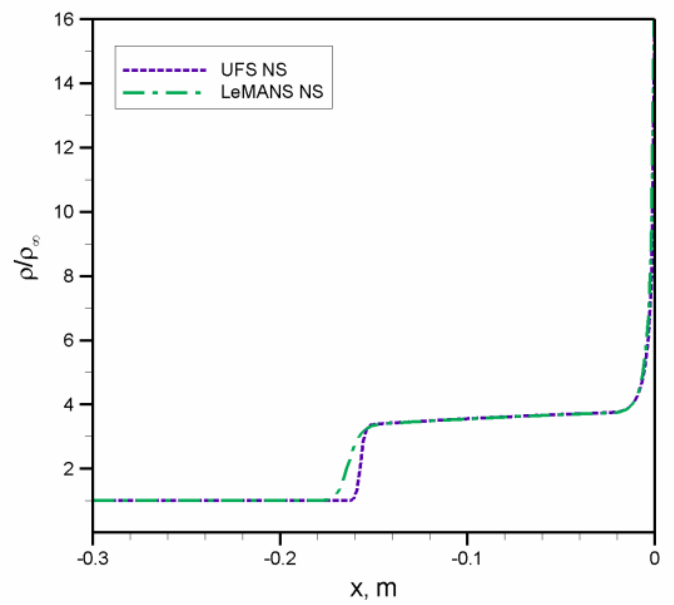

Figure 30. Density along the stagnation streamline for $K n=0.003$. 


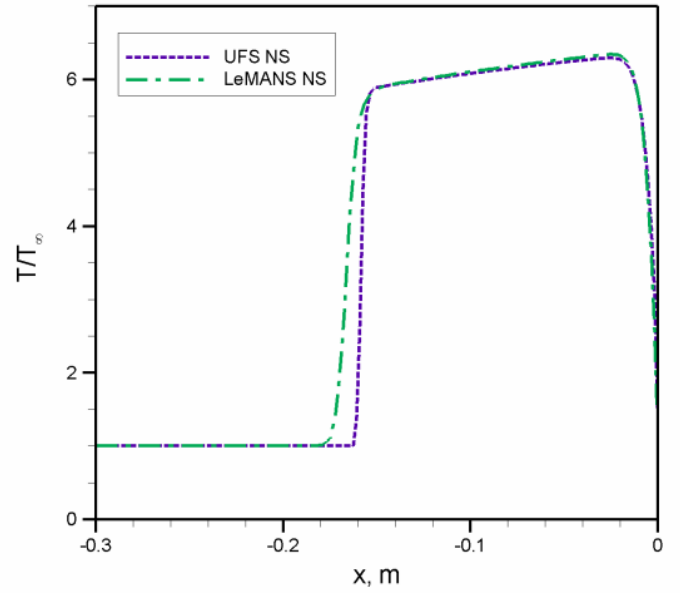

Figure 31. Temperature along the stagnation streamline for $\mathbf{K n}=\mathbf{0 . 0 0 3}$.

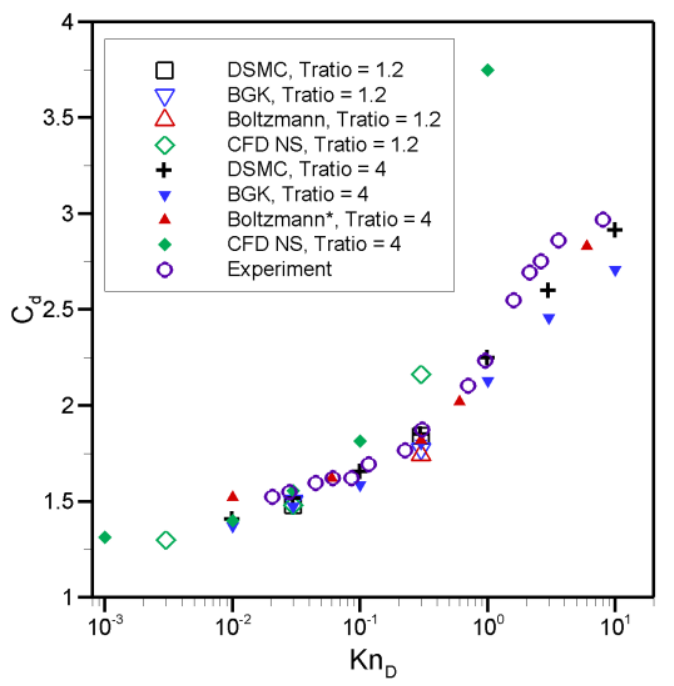

Figure 32. Variation in drag coefficient with Knudsen number. 